Special issue of the 2nd International Conference on Computational and Experimental Science and Engineering (ICCESEN 2015)

\title{
Adequate Method for Decoupling Bulk Lifetime and Surface Recombination Velocity in Silicon Wafers
}

\author{
N. Khelifati ${ }^{a, b, *}$, D. Bouhafs ${ }^{a}$, A. Mebarek-Azzem $^{c}$, S. El-Hak Abaidia $^{b}$, \\ B. PAlahounane ${ }^{a}$ And Y. Kouhlane ${ }^{a}$ \\ ${ }^{a}$ Centre de Recherche en Technologie des Semi-conducteurs pour l'Energétique (CRTSE), DDCS, Algiers, Algeria \\ ${ }^{b}$ Université M'hamed Bougara (UMBB), Faculté des Sciences, Boumerdes, Algeria \\ ${ }^{c}$ Université de Sétif, Faculté des sciences-Département de physique, Sétif, Algeria
}

\begin{abstract}
In this paper, we present an appropriate method of decoupling surface and bulk recombination processes in silicon wafers. The study was carried out using the surface passivation of multicrystalline silicon wafers by ethanolic solution of iodine at different molarities varying between $0.01 \mathrm{M}$ and $0.1 \mathrm{M}$. The effect of the concentration of ethanolic iodine solution on surface passivation effectiveness was investigated by using quasi steady state photoconductance technique. Reproducible experiments have shown that the best passivation is reached for a molarity of around $0.02 \mathrm{M}$. The carrier lifetime after passivation at $0.02 \mathrm{M}$ has been improved by more than one order of magnitude, compared to that of the same wafer before the passivation. Using an adequate modeling of minority carrier lifetime curves $\tau(\Delta \mathrm{n})$, based on Hornbeck-Haynes model, surface recombination velocity was calculated. The minimum values of surface recombination velocity have been found to be approximately $120 \mathrm{~cm} / \mathrm{s} \mathrm{for} 0.02 \mathrm{M}$. The modeling results indicate that the minority carrier lifetime improvement can be easily correlated with the decrease of the surface recombination velocity for a fixed bulk lifetime $\tau_{b}=115 \mu \mathrm{s}$.
\end{abstract}

DOI: 10.12693/APhysPolA.130.188

PACS/topics: 68.47.FG, 88.40.H-

\section{Introduction}

In semiconductor and solar cell technologies the inspection of the bulk minority carrier lifetime $\tau_{b}$ is a crucial step for monitoring the material quality, determination of the cleanliness of furnaces, and establishing the effects of various process steps during device processing [1]. However, a reliable measurement of $\tau_{b}$ is not easy and involves careful preparation of the wafer. One of the essential requirements for an appropriate measurement of $\tau_{b}$ is that the wafer surfaces and edges be well cleaned and passivated, especially in the case of wafers that have higher electrical quality.

Currently, two main techniques are used by the photovoltaic community to inspect the electrical quality of silicon wafers; microwave photoconductance decay $(\mu$ PCD) and quasi steady state photo-conductance technique (QSSPC). In spite of the fact that the $\mu-\mathrm{PCD}$ is an effective technique to study the carrier lifetime distribution, the QSSPC still remains an advantageous technique in terms of simplicity of use and short measurement time. Nevertheless, since recombination occurs in the bulk as well as on both surfaces of the wafers, the measured lifetime is an effective value $\tau_{\text {eff }}$ that has contributions from the bulk $\tau_{b}$ and the two surfaces $\tau_{s}$. It can be described by

$$
\frac{1}{\tau_{\mathrm{eff}}}=\frac{1}{\tau_{b}}+\frac{1}{\tau_{s}},
$$

where

\footnotetext{
* corresponding author; e-mail: n.khelifati@gmail.com
}

$$
\tau_{s}=\frac{W}{2 S}+\frac{1}{D_{n}}\left(\frac{W}{\pi}\right)^{2}
$$

$S$ is the surface recombination velocity, $W$ is the wafer thickness and $D_{n}$ is the diffusion constant of minority carriers.

When the surface undergoes a good passivation; $\tau_{\text {eff }} \approx \tau_{b}$. The recombination activity can be reduced either through thin films deposition such as $\mathrm{SiO}_{x}, \mathrm{Si}_{x} \mathrm{~N}_{y}$, $\mathrm{Al}_{2} \mathrm{O}_{3}$, a-Si:H and a-SiC:H or by formation of a floating $\mathrm{N} / \mathrm{P}$ junction, or by immersion in chemical solution using hydrofluoric acid (HF) and iodine in ethanol or methanol (I-E or I-M) solution. The first kind of passivation techniques requires an important thermal budget that can't be avoided [2, 3], accompanied by bulk properties modification more or less significant. Therefore, chemical passivation route seems to be fast, simple, not expensive and more adequate to inspect the bulk quality and then to predict the final solar cell efficiency. Chhabra et al. [4] and Swain et al. [5] have, respectively, used quinhydrone- and iodine-methanol to passivate silicon and germanium, and have obtained high passivation qualities. Another study carried out by Stephens and Green [3] demonstrated that good surface passivation can be achieved using $0.08 \mathrm{M}$ iodine-ethanol solution.

In the present work, we report a systematic study of decoupling of bulk lifetime and surface recombination velocity in silicon wafers. It refers to a semi-empirical method based on the modeling of experimental results obtained by QSSPC.

\section{Experimental details}

A set of five adjacent gettered wafers (P6, P7, P8, $\mathrm{P} 9$ and $\mathrm{P} 10$ ) selected from the top region of ingot were 
used in this study. The wafers were $1.5 \Omega \mathrm{cm}$, p-type, of multicrystalline silicon, grown by the heat exchanger method [6], with thickness of $300 \mu \mathrm{m}$. Firstly, wafers were chemically etched in $\mathrm{NaOH}: \mathrm{H}_{2} \mathrm{O}(30 \%)$ at $80^{\circ} \mathrm{C}$, followed by dipping in $\mathrm{HF}(10 \%)$ and rinsing in deionized water. Later on, wafers were placed in transparent polyethylene bags without I-E solution, and lifetime measurements were directly performed by QSSPC technique (Sinton Consulting, WCT-120 tester). The obtained results are considered as references (Ref.). After this step, the five wafers were passivated by adding the same concentration of I-E into the bags containing the wafers, and then the lifetime measurements were done again. Subsequently, the wafers were dipped in diluted $\mathrm{HF}$, dried and passivated using another concentration of I-E. The cleaning-passivation-measure process was repeated for different I-E solution concentrations ranging from $0.01 \mathrm{M}$ to $0.1 \mathrm{M}$.

\section{Results and discussion}

Figure 1 illustrates the evolution of measured minority carrier lifetime $\tau$ vs. injection level $\Delta n$ curves obtained for wafer $\mathrm{P} 6$ without and with passivation at different I-E concentrations. We note that similar results were found for the rest of studied wafers (P7, P8, P9 and P10).

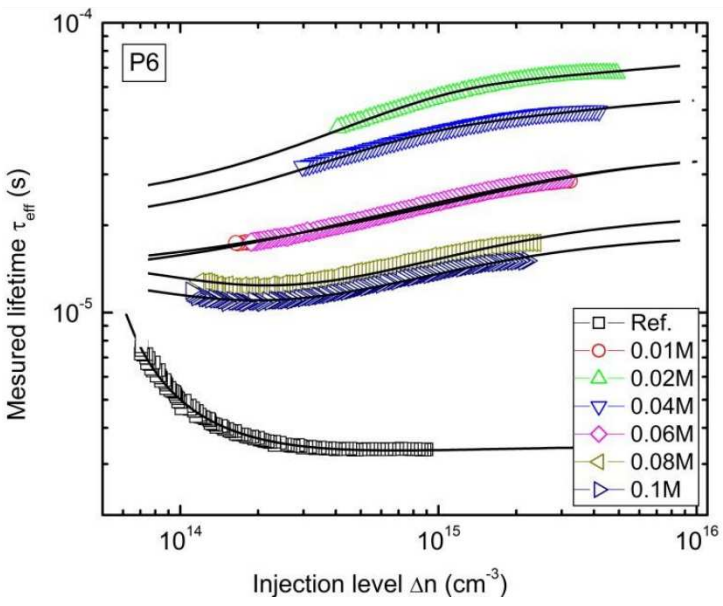

Fig. 1. Evolution of injection level dependent measured lifetime curves, obtained by QSSPC technique for wafer P6 after passivation at different concentrations of I-E solution. Solid lines represent fits made using Hornbeck-Haynes model.

We observe that I-E concentration has a notable effect on $\tau(\Delta n)$ curves. This effect can be clearly shown by the increase of the measured lifetime and also the shift of curves towards higher injection level $\Delta n$. This evolution is accompanied by a change in the curves slop, especially before and after the I-E passivation. Lifetime simulation demonstrates that the decrease of surface recombination velocity yields similar evolution of lifetime curves. Such observation leads us to assumption that the evolution of $\tau(\Delta n)$ is probably due to an effective surface passivation.
For checking quantitatively this assumption, an adequate modeling of $\tau(\Delta n)$ has been done using Hornbeck-Haynes model [7-9]. In our previous study [10], the same model was employed to investigate bulk lifetime improvement after the gettering process.

During the modeling of lifetime curves, bulk parameters such as recombination centre density $N_{r}$ and capture cross-sections $\sigma_{n}$ and $\sigma_{p}$ were carefully computed and fixed at appropriate values for all studied wafers (see Table I). The only parameter which has been considered variable is the surface recombination velocity (SRV). In the present study, SRV was assumed independent of injection level $\Delta n$.

TABLE I

Appropriate bulk parameters $\left(N_{r}, \sigma_{n}\right.$ and $\left.\sigma_{p}\right)$ used in modeling and the resulting bulk lifetime $\tau_{b}$.

\begin{tabular}{c|c|c}
\hline \hline Bulk parameters & Computed values & Bulk lifetime $[\mu \mathrm{s}]$ \\
\hline $\mathrm{N}_{r}$ & $8 \times 10^{11} \mathrm{~cm}^{-3}$ & \multirow{2}{*}{115} \\
\hline$\sigma_{n}$ & $2 \times 10^{-14} \mathrm{~cm}^{2}$ & \\
\hline$\sigma_{p}$ & $3 \times 10^{-16} \mathrm{~cm}^{2}$ &
\end{tabular}
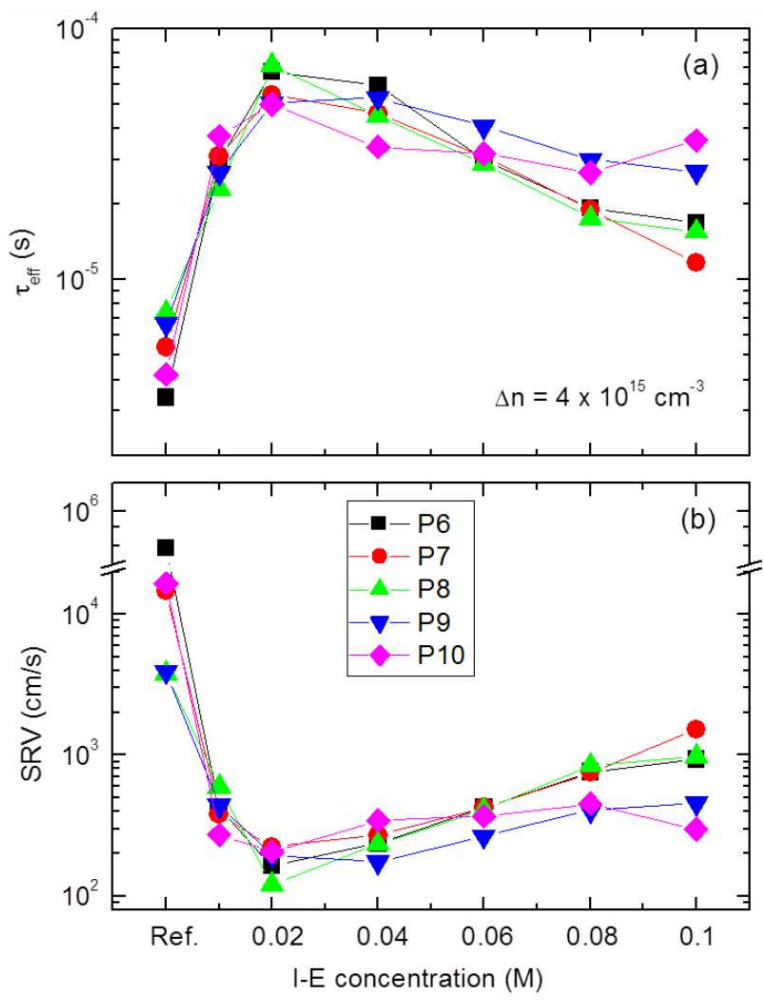

Fig. 2. Variation of the effective lifetime of all studied wafers (a) and the associated surface recombination velocity (b) as function of concentration of the I-E solution.

The effect of I-E solution concentration on the effective lifetime and SRV, estimated by fitting, is presented in Fig. 2a and b, respectively. We observe an obvious reproducibility of passivation experiment, where all wafers showed a similar lifetime variation with I-E 
concentration. The lifetime reached maximum values for I-E concentration of around $0.02 \mathrm{M}$, with an increment of $\sim 900 \%$ compared to the lifetime measured without passivation. This increment of lifetime is accompanied by a clear degradation of calculated SRV. The minimum SRV $(\sim 120 \mathrm{~cm} / \mathrm{s})$ is reached at $0.02 \mathrm{M}$. Correlation between experimental and modelling results leads us to conclusion that the lifetime improvement is due to an effective surface passivation. Same inverse variation of SRV with lifetime can be observed beyond $0.02 \mathrm{M}$, confirming the above explanation.

Figure 3 summarizes the obtained results for all studied wafers; P6, P7, P8, P9 and P10. It shows the variation of effective lifetime $\tau_{\text {eff }}$ measured by QSSPC at $\Delta n=4 \times 10^{15} \mathrm{~cm}^{-3}$ as function of SRV determined by fitting. The solid line represents an appropriate theoretical curve $\tau_{\text {eff }}$ (SRV) plotted employing Eqs. (1) and (2). The bulk lifetime $\tau_{b}=115 \mu$ s used in plotting the curve was calculated from bulk parameters (see Table I). This figure shows clearly a good consistency between the experimental data and modeling findings. It demonstrates also a reliable decoupling of SRV and $\tau_{b}$ for all measured values of $\tau_{\text {eff }}$.

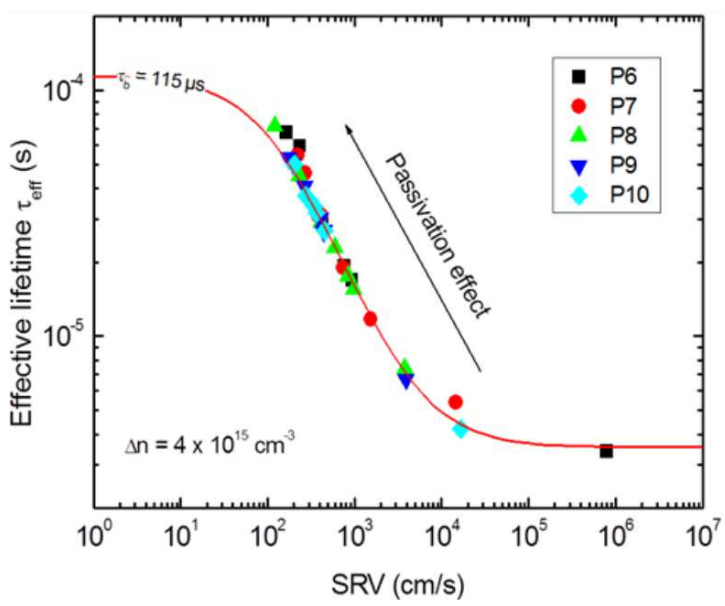

Fig. 3. Variation of the effective lifetime $\tau_{\text {eff }}$ as function of surface recombination velocity SRV obtained for all studied wafers. Solid line is the theoretical plot of $\tau_{\text {eff }}(\mathrm{SRV})$ for $\tau_{b}=115 \mu \mathrm{s}$.

\section{Conclusions}

In the present work, we report a systematic investigation of surface passivation made on p-type multicrystalline silicon wafers using different iodine-ethanol molar concentrations. Measurements of the minority carrier lifetime $\tau$ were made for iodine-ethanol molarity varying from $0.01 \mathrm{M}$ to $0.1 \mathrm{M}$. Reproducible experiments showed an effective surface passivation at the optimal I-E molarity of $0.02 \mathrm{M}$. At this particular molarity the surface recombination velocity was estimated by modeling to be around $120 \mathrm{~cm} / \mathrm{s}$, and the bulk lifetime was found to be equal to $115 \mu \mathrm{s}$ (at $\Delta n=4 \times 10^{15} \mathrm{~cm}^{-3}$ ). This study has demonstrated the potential of the used method for decoupling surface recombination velocity and bulk lifetime from the measured effective lifetime.

\section{References}

[1] B. Sopori, S. Devayajanam, P. Basnyat, V. Mehta, H. Moutinho, B. Nemeth, V. LaSalvia, S. Johnston, N.M. Ravindra, J. Binns, J. Appel, in: Symposium $E / H-$ Advances in the Characterization, Performance and Defect Engineering of Earth Abundant and Thin-Film Materials for Solar Energy Conversion, MRS Proceedings, Vol. 1670, Materials Research Society, 2014, p. mrss14-1670-h05-02.

[2] S. Aouida, N. Bachtouli, B. Bessais, Appl. Surf. Sci. 274, 255 (2013).

[3] A.W. Stephens, M.A. Green, Sol. Energ. Mater. Sol. Cells. 45, 255 (1997).

[4] B. Chhabra, S. Bowden, R.L. Opila, C.B. Honsberg, Appl. Phys. Lett. 96, 063502 (2010).

[5] B.P. Swain, H. Takato, I. Sakata, Appl. Phys. Express 2, 105501 (2009).

[6] D. Ouadjaout, Y. Gritli, L. Zair, M. Boumaour, Revue des Energies Renouvelables 8, 49 (2005).

[7] J.A. Hornbeck, J.R. Haynes, Phys. Rev. 97, 311 (1955).

[8] J.R. Haynes, J.A. Hornbeck, Phys. Rev. 100, 606 (1955).

[9] A. Bentzen, Ph.D. Thesis, University of Oslo, 2006.

[10] N. Khelifati, D. Bouhafs, M. Boumaour, Mat. Sci. Semicon. Proc. 15, 56 (2012). 